\title{
A Way of Candidate Set Selection for Epidemic Routing in Delay Tolerate Networks
}

\author{
Fuquan Zhang ${ }^{1 *}$, Kaiqi Chen, Inwhee Joe ${ }^{2}$,Demin Gao ${ }^{1}$ and Yunfei Liu ${ }^{1}$ \\ ${ }^{1}$ College of Information and Sciences \\ NanJing Forestry University \\ NanJing, China \\ ${ }^{2}$ Department of Electronic and Computer Engineering \\ Hanyang University \\ Seoul, Korea \\ E-mail: zfq@njfu.edu.cn; 546285799@qq.com; iwjoe@hanyang.ac.kr; \\ dmgao@njfu.edu.cn;
}

\begin{abstract}
The radio propagation effect of fading or shadowing between mobile nodes has not been well considered in contact rate. One way to incorporate interference into contact rate is multiplied by probability that the infected node copies the message to the susceptible node during a contact in epidemic routing. In this paper, we develop a model to incorporate the wireless link reliability into contact rate. For application, we proposed a scheme for epidemical delay tolerate network with considering the effects based on the model. Simulation shows that proposed scheme has better performance.
\end{abstract}

Keywords: epidemiological model; channel impairments; delay tolerate network; epidemic

\section{Introduction}

The SI (Susceptible-Infected) is a classical epidemiological model that developed to model the spread of the epidemic in population. Several mathematical models of epidemical DTN (Delay Tolerant Networks) are based on the SI model due to the similarity between the message forwarding in a DTN and epidemic spreading in population [1-2]. The performance of wireless communication systems is influenced by the radio propagation effect in mobile channel. The effect not only leads to signal attenuation with the propagation distance but also yields a large fluctuation of signal strength within a short range

Channel impairments can be considered into a parameter that is called contact rate, which is defined as the average number of contacts between two nodes per unit time. Paper [4] proposed a probabilistic routing that considers attenuating the contact rate with a multiplicative constant to take into account the decision of the forwarding node. This attenuated contact rate is called effective contact rate, and represents the average number of contacts between a pair of nodes per unit time in which a message is successfully transmitted. One way to incorporate interference into contact rate is multiplied by probability that the infected node copies the message to the susceptible node during a contact [5].

The radio propagation effect of fading or shadowing between mobile nodes has not been well considered in contact rate. Moreover, characteristics of a mobile-tomobile radio channel are quite different compared with the traditional stationary base station to mobile radio channel. Mobile to mobile radio channels can be found in ad-hoc networks, sensor networks, vehicle-to-vehicle communications and DTN. 
The wireless radio channel poses a severe challenge as a medium for reliable communication. Not only is it susceptible to noise and channel impediments, but these impediments change over time as a result of user movement and environment dynamics [3].

During the recent years, statistical properties of mobile-to-mobile channels have been investigated by many researchers concentrating on small-scale characteristic of the mobile-to-mobile radio channels [6-7]. These parameters of the derived model should be determined by carrying out measurements in the area where the mobileto-mobile system will be operated.

The typical DTN protocols can be classified into single-copy and multi-copy routing protocols. They represent a natural trade-off in DTN networks.

Single-copy protocol forward messages to the first node they encounter or final destination, which results in a "random walk" search for the destination node and only a single copy of every message in the network. Multi-copy protocol, such as Epidemic routing [8], in contrast, forwards all non-duplicated messages, including its own, to every node it encounters - eventually delivering its messages to the appropriate destinations.

ANEpidemic uses angle and position information of local node to control the ineffective duplication messages [9]. It selects next hops of messages through judgments of relative positions between nodes (node that is carrying message and the node that will relay the message to destination node.). ANEpidemic thinks that a node that is closer to the destination node, the greater probability of the node that may meet the destination by stochastic motion. The node that is carrying message is as the center of a circle, and spreading range of the node as its radius. ANEpidemic selects nodes that are closer to the destination node as the next hop of messages in the semicircle with angle $90^{\circ}$.

In this paper, by considering radio propagation effect on signal strength, wireless radio link reliability of mobile nodes is incorporated into effective contact rate. For application, a candidate set (CS) scheme for message forwarding in DTN with epidemic routing is proposed, which considers contact rate estimation to some extent without compromising on simplicity of epidemical algorithm. The performance of proposed scheme is compared with related algorithm. Simulation shows that CS has better performance.

The remainder of this paper is organized as follows. In Section 2, the link reliability estimation for contact rate is described. Section 3 proposed an forwarding scheme. The simulation results are presented in Section 4. Conclusion is in Section 5 .

\section{Link Reliability Estimation for Contact Rate}

Two critical tasks are completed for incorporating wireless radio link reliability of mobile nodes in contact rate including:

(1) Modeling of mobile-to-mobile path loss.

(2) Proposing an effective contract rate estimation method.

\subsection{Proposed Mobile-to-Mobile Path Loss Model}

A base-to-mobile power law path loss model was selected to predict the mobileto-mobile channels communication over distance [3].

In order to validate that this base-to-mobile model works well for mobile-tomobile channels, and to determine the parameters' values for application, we carried out experiments in spring at rural area in xi-an city, China. The measurement parameters are summarized in Table 1. The transmitter antennas and the receiver 
antennas were omni-directional antennas. The antennas and a spectrum analyzer were mounted on the cars with height of 1.5 meters. A continuous wave signal of $914 \mathrm{MHz}$ carrier frequency was fed to the transmitter antenna. [6][7].

Table 1. Measurement Configuration

\begin{tabular}{|c|c|}
\hline Parameters & Values \\
\hline Transmitter & PCS-20 \\
\hline Receiver & FSL6 Spectrum Analyzer \\
\hline TX Antenna & $3.0 \mathrm{dBi}$ \\
\hline RX Antenna & $3.0 \mathrm{dBi}$ \\
\hline Frequency & $914 \mathrm{MHz}$ \\
\hline Antenna Height & $1.5 \mathrm{~m}$ \\
\hline Antenna Height & $1.5 \mathrm{~m}$ \\
\hline Feeder Loss & $2.9 \mathrm{~dB}$ \\
\hline Cable Loss & $2.0 \mathrm{~dB}$ \\
\hline
\end{tabular}

$P L(d)$ is the mean path loss value, $d$ represents the distance from the transmitter.

$P L\left(d_{0}\right)$ is the reference path loss at distance $d_{0}$. Variable $n$ is the path loss exponent and it describes how quickly the signal attenuates. Where $N(0, \sigma)$ follows a zero-mean Gaussian distribution with a variance $\sigma$ to reflect effects of shadowing and fading [10-11].

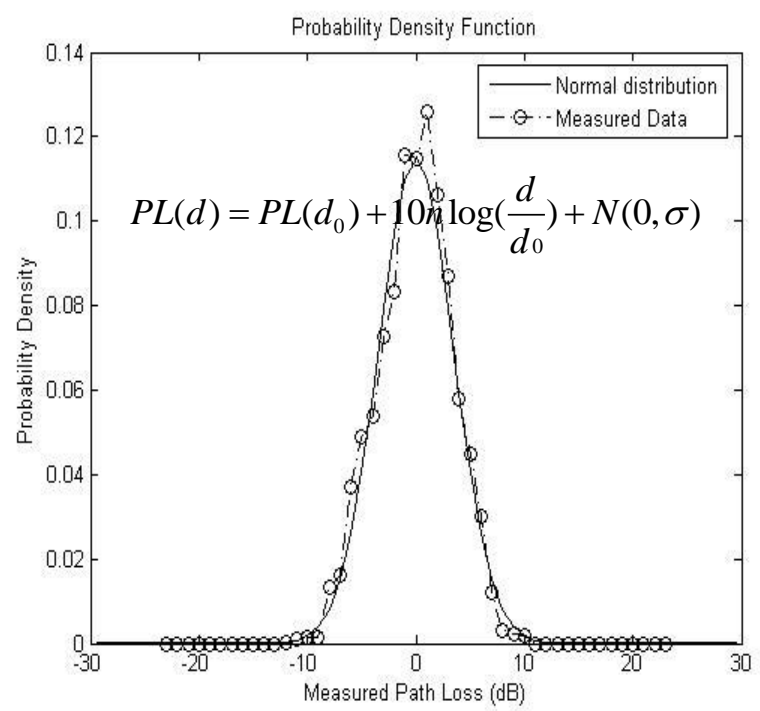

(1)

\section{Figure 1. The Measured Pat Loss (dB) at $914 \mathrm{MHz}$ is Curve-Fitted by a Normal Distribution with a given Standard Deviation $\sigma$ Value 3.47in Rural Area}

Figure 1 shows the measured path loss rural area. The solid curve shows the normal distribution with standard deviation. The measured Probability Distribution Function (PDF) has actually been shown by the dashed line. The measured $\mathrm{n}$ is 3.6, and a zero-mean normal distribution with a given standard deviation $\sigma 3.47$ in rural area. The results are similar to the results that measured in other rural mobile-tomobile scenarios [8][12]. 


\subsection{Proposed Effective Contract Rate}

Contact rate is defined as the average number of contacts between two nodes per unit time. The SI (Susceptible-Infected) is a classical epidemiological model that developed to model the spread of the epidemic in population [5]. Any individual can be classified into susceptible or infected states. Mobile nodes are treated in the same way as individuals in a population when the SI model is applied to the DTN. Therefore, an infected node forwards a copy of the message to each susceptible node that it contacts. Several mathematical models of epidemical DTN are based on the SI model due to the similarity between the message forwarding in a DTN and epidemic spreading in population [2], [5]. Assuming that DTN nodes move in an area L2, an estimative for the contact rate [5][8] of epidemical DTN is

$$
\beta \approx \frac{2 w E\left[v^{*}\right]}{L^{2}} d_{t x}
$$

Where $\mathrm{w}$ is a constant specific to mobility model, $d_{t x}$ is the transmission range of nodes and $E\left[v^{*}\right]$ is the average relative speed between two nodes.

Paper [4] proposed a probabilistic routing that considers attenuating the contact rate with a multiplicative constant to take into account the decision of the forwarding node. Interference is incorporated into contact rate by multiplying the probability that the infected node copies the message to the susceptible node during a contact [5]. This attenuated and incorporated contact rate are called effective contact rate, and represents the average number of contacts between a pair of nodes per unit time in which a message is successfully transmitted [5].

One way to incorporate link reliability into the contact rate is considering that the infected node copies the message to the susceptible node with some probability $\mathrm{p}$ during a contact [5]. Thus, it would be enough to replace $\beta$ by $\beta p_{l}$ in (2) so that the effects of link reliability are considered [8]. We defined $\beta p_{l}$ as effective contact rate that represents the average number of contacts between two nodes per unit time [4$5]$, in which an infected node successfully transmits a message to a susceptible one[12].

$$
\beta p_{t}=\frac{2 w E\left[v^{*}\right]}{L^{2}} d_{t x} p_{l}
$$

\subsection{Estimation of Link Reliability}

In wireless systems there is typically a target minimum received power level $\mathrm{P}_{\text {min }}$ below which performance becomes unacceptable (e.g., the voice quality in a cellular system becomes too poor to understand) [1]. The transmission range, dtx, should be a distance so that the received power at the susceptible node is greater than the minimum received power.

In the proposed scheme, the link reliability $\mathrm{pl}$ for a radio link is defined to be the probability that the received power at a given distance $d_{t x}, P_{r}\left(d_{t x}\right)$, falls above $P_{\min }$ :

$$
L R_{i}=p\left(P_{r}\left(d_{i}\right) \geq P_{\min }\right)
$$

With Eq.(2) and some derivations [1][9], LRi is given by 


$$
L R_{i}=Q\left(\frac{P_{\min }-\left(P_{t}-P L\left(d_{0}\right)-10 n \log \left(\frac{d_{i}}{d 0}\right)\right)}{\sigma}\right),
$$

where $Q(z)$ is a complementary error function and is defined as:

$$
Q(z)=\int_{z}^{\infty} \frac{1}{\sqrt{2 \pi}} e^{-x^{2} / 2} d x .
$$

A radio link with a higher LRi value can be considered better than others in channel of network, and can be used with a first for path selection in routing discovery.

The parameters $P_{t}$ can be configured based on transmission power. The $P_{\min }$ can be configured based on target minimum received power. It is noted that the path loss exponent $\mathrm{n}$ and the variance $\sigma$ should be adaptive to the propagation environment of the application [11].

\section{Proposed Scheme}

We assume that the distance between the neighbor nodes at time instance $t_{i}$ is $d_{i}$, as shown in Figure2.a. We assume that each nodes knows their location. We assume that the nodes move in different directions at constant speed $v_{1}$ and $v 2$ respectively, as shown in Figure2.b. During a time interval $T$, the distance $d_{i+1}$ between two neighbor nodes at time $t_{i+1}$ is:

$$
d_{i+1}=\sqrt{\left[\left(v_{2} * \operatorname{Sin} \theta_{2}-v_{1} * \operatorname{Sin} \theta_{1}\right) * T\right]^{2}+\left[\left(v_{1}{ }^{*} \operatorname{Cos} \theta_{1}+v_{2} * \operatorname{Cos} \theta_{2}\right) * T+d_{i}\right]^{2}}
$$

If the nodes keep their original directions and move exactly inversely Equation 7 gets the maximum value of the Expression 8. (that is, on the extension line of $d_{i}$ ). Thus, the received power at the susceptible node will be lower than the minimum received power as shown in Figure2.

$$
d_{i+1}=d_{i}+\left(v_{1}+v_{2}\right) * T
$$

If the nodes move the same direction along the $d_{t x}$, Equation 7 will get its minimum value Expression 9.

$$
d_{i+1}=d_{i}-\left|v_{1}-v_{2}\right| * T
$$

Since $d_{t x}$ should be a distance so that the received power at the susceptible node is greater than the minimum received power. In order to satisfy the requirement on the minimum received power, the copy and distribution of message can be decreased by considering the movement direction of nodes [8]. That is, the invalid replication of message should be reduced by utilizing movement and direction information. 


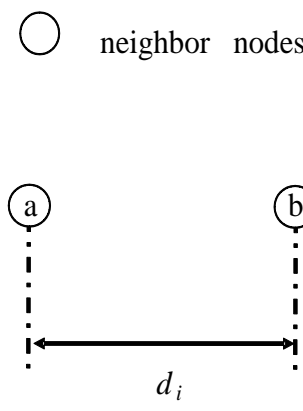

at $i^{\text {th }}$ time interval

(a)

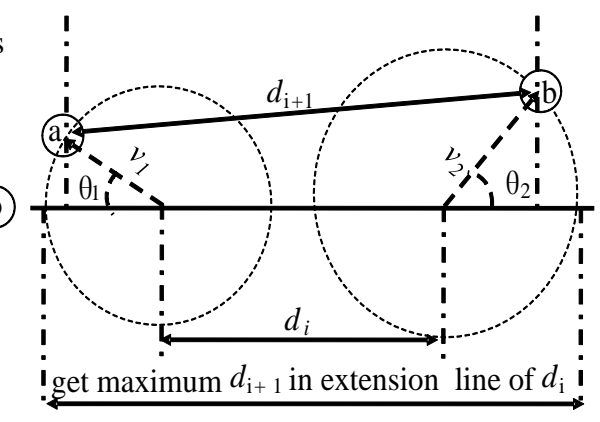

at $(i+1)^{\text {th }}$ time interval

(b)

Figure 2. Factors that Affect Relaying Messages

This allows the intermediate nodes to relay messages based on movement and direction information, and helps to reduce overhead to some extent.

For a fixed time interval, the contact rate is affected by node velocities and direction. Thus, estimation of contact rate can be a function of the node velocity and direction. Moreover, the forwarding strategy needs to be chosen carefully to avoid invalid duplication of message and reduce burdens on calculation.

In this paper, we proposed a candidate set (CS) routing strategy to consider contact rate without compromising on simplicity of epidemical algorithm.

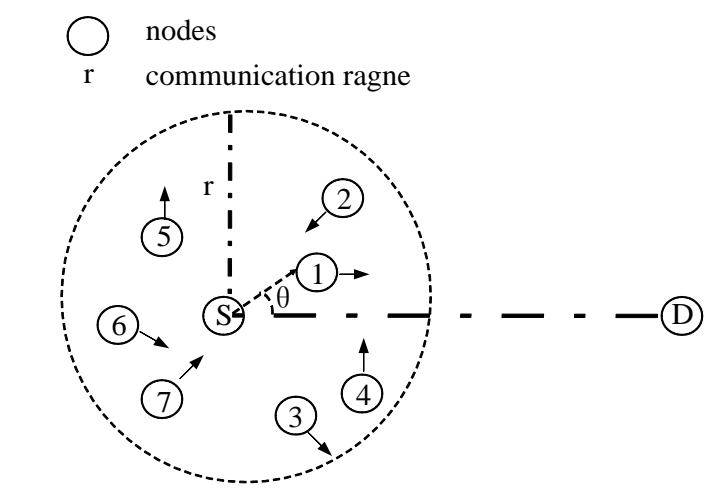

Figure 3. Strategy used in the Scheme

For each node $\mathrm{s}$, let $\mathrm{N}(\mathrm{s})$ be the set of its one-hop neighbors and let $\mathrm{D}(\mathrm{s})$ be the set of all distances of $s$ from all its hop-one neighbors $v \in N(s)$. Neighbors can be detected and distances can be measured using radio-location. Each node $\mathrm{s}$ knows all its one-hop neighbor $\mathrm{N}(\mathrm{s})$ and distance $\mathrm{D}(\mathrm{s})$ sets to all its one-hop neighbors. The candidate nodes at $\mathrm{s}$ can be selected as follows. Node $\mathrm{s}$ selects the nodes in its onehop neighborhood $\mathrm{N}(\mathrm{s})$ in such a way that their distance dtx should be satisfied so that the received power at the susceptible node is greater than the minimum received power.

The candidate set is initially established by considering all possible nodes and selecting the best $\mathrm{k}$, where $\mathrm{k}$ is the size of the set, based on their moving velocity or direction values. This set is then used for the next 1 iterations of the algorithm.

Here, we choose the nodes that can minimize the initially angle $\theta$ and the nodes that are moving toward to the extension line of S-D as candidate set. In this case they are node 4 and node 1, as shown in Figure3. 


\section{Simulation Results}

To evaluate and compare the effectiveness of the proposed path stability evaluation method, we performed simulations in NS-2.32 [13]. Each simulation is carried out under different mobility and traffic pattern to obtain the average value. To include random characteristics of mobile-to-mobile radio channels, the path loss models with a path loss exponent $n=3.6$ and a variance of $\sigma=3.47$ was chosen.

\subsection{Simulation Parameters}

The simulation parameters are presented in Table 2 .

Table 2. Simulation Parameters

\begin{tabular}{|c|c|}
\hline Parameters & Values \\
\hline Simulation time & $21600 \mathrm{~s}$ \\
\hline Buffer size & $35 \mathrm{M}$ \\
\hline Wait time & $0-120$ \\
\hline Maximum peed & $5 \mathrm{~m} / \mathrm{s}$ \\
\hline Message TTL & $18000 \mathrm{~s}$ \\
\hline Mobile Model & MBM, RWP \\
\hline Transmit speed & $2 \mathrm{Mbps}, 10 \mathrm{Mbps}$ \\
\hline Event generators & $30 \mathrm{~s}-60 \mathrm{~s}$ \\
\hline Protocols & $\mathrm{CS}, \mathrm{ANEpidemic}$ \\
\hline Message sizes & $1 \mathrm{MB}$ \\
\hline Number of Nodes & 480 \\
\hline
\end{tabular}

\subsection{Performance Metrics}

Performance metrics were used in this paper as follows:

(1) Delivery Probability is the ratio of data packets being successfully received by the destination nodes versus data packets being sent by the source nodes.

(2) Overhead Ratio is defined as:

$$
\text { overhead ratio }=\frac{\text { number of relayed messages }- \text { number of delivered messages }}{\text { number of delivered messages }}
$$

It's some measure of how much "overhead" there was in relation to delivered messages[12].

\subsection{Varying Speed}

The maximal node speed was increased from $1.5 \mathrm{~m} / \mathrm{s}$ to $4.5 \mathrm{~m} / \mathrm{s}$. As can be observed from Figure 4a, increasing mobility improves the delivery rate of all the protocols due to the increasing probability of encountering potential destination node. As mobility increases, more nodes will encounter new node, and high chance of duplicating message to encounter potential destination to some extent.

As shown in Figure4b, routing overhead is sensitive to the speed of nodes when it increases. The probability of encountering new nodes is gradually increasing when the speed is increasing, which resulting in a large message exchange. The overhead rate is decreasing due to the increasing in delivery rate. 


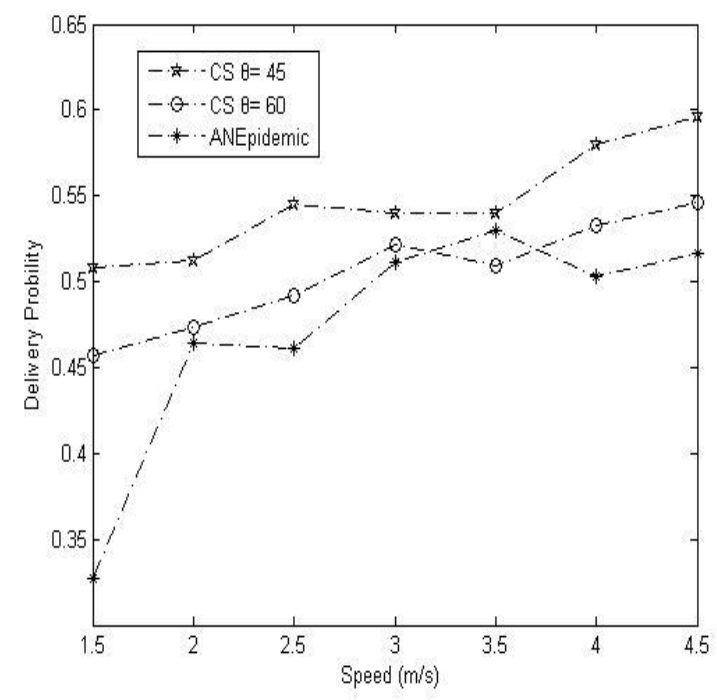

(a) delivery

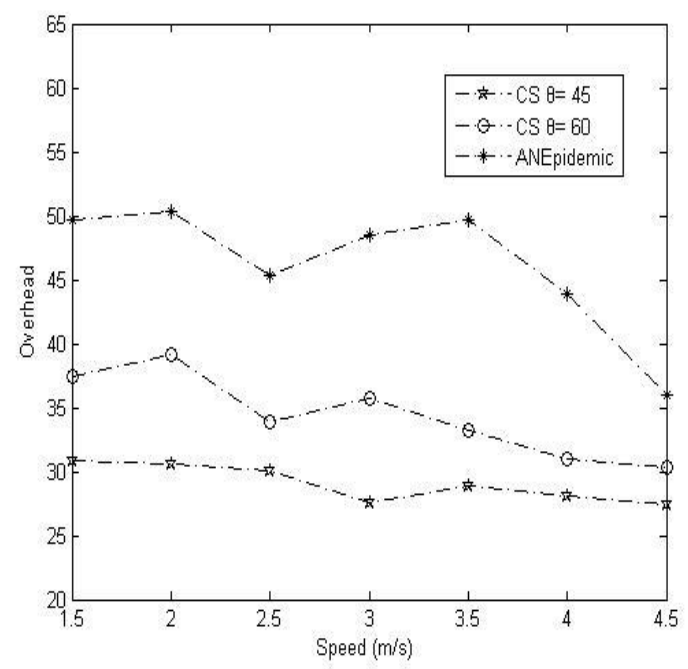

(b) overhead rate

Figure 4. Varying Mobile Speed

\subsection{Varying Number of Nodes}

The difference of all protocols is not very noticeable while varying the number of nodes in delivery rate. With increase in the nodes, CS tends to perform better relatively, as shown in Figure 5a. As shown in Figure5b, routing overhead is gradually increasing when the number of node increases. The probability of encountering new nodes is increasing when there is more nodes in the network. Thus, the overhead rate is increasing with the increasing in the number of nodes. 


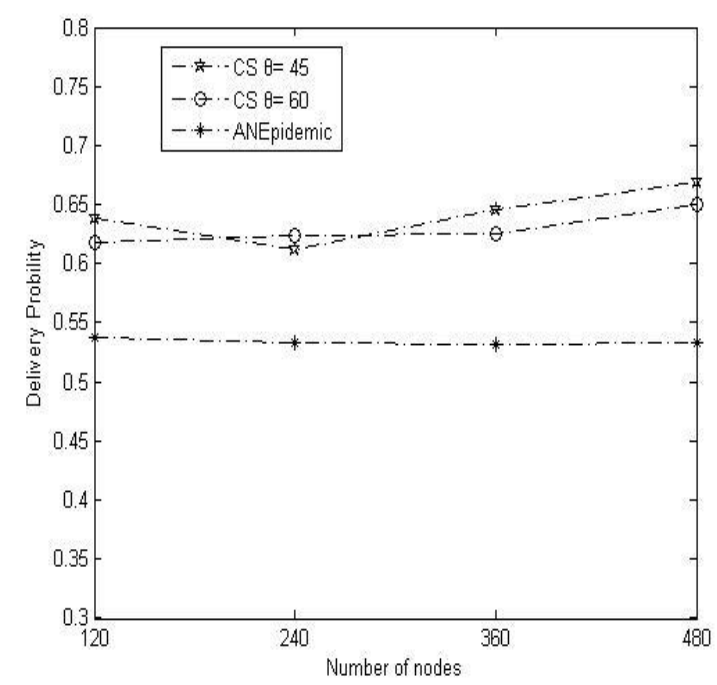

(a) delivery

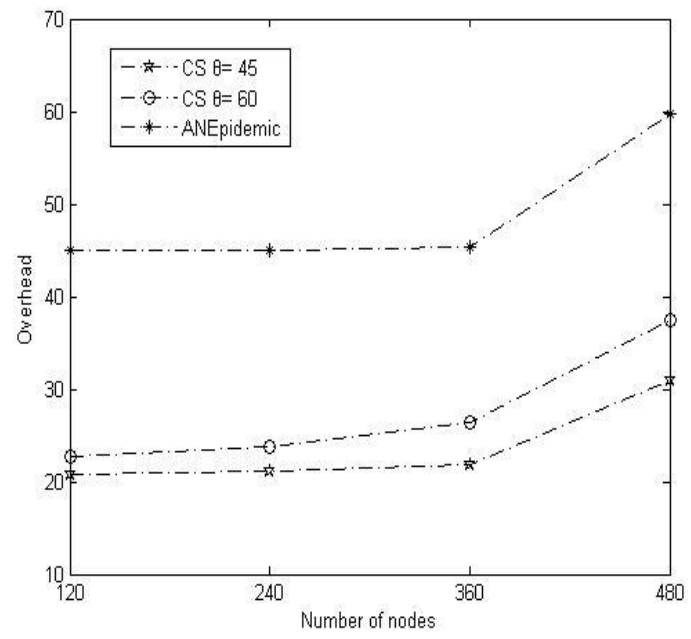

(b) overhead rate

Figure 5. Varying Node Number

\section{Conclusion and Future Work}

The signal strength of wireless communication medium may vary over distance and environment where the mobile nodes are. In this paper, we develop a model to incorporate the wireless link reliability into contact rate. Here, the received signal strength between adjacent nodes is predicted by a validated mobile-to-mobile radio propagation model.

For application, we proposed a scheme for delay tolerate network with considering the effects based on the model. These parameters were derived from a base-to-mobile path loss model by real mobile-to-mobile environment. Simulation shows that proposed scheme has better performance. 


\section{Acknowledgments}

This work was supported by TAPP:Top-notch Academic Programs Proects of Jiangsu Higher Education Institutions. The project sponsored by the Scientific Research Foundation for the Returned Overseas Chinese Scholars, State Education Ministry. This work was supported by the Foundation of NanJing Forestry University,G2014105. This work was supported by Natural Science Foundation of Jiangsu Province, BK20150880. The corresponding author is fuquan zhang.

\section{References}

[1] A. Goldsmith, "Wireless Communications", Cambridge University Press, Reprinted, (2007).

[2] G. M. S. Dias and R. M. Salles, "Epidemic SIR model applied to delaytolerant networks", In Proceedings 30th Brazilian Telecommun. Symp, (2012), pp. 1-5.

[3] C. Abreu and R. Salles, "Modeling message diffusion in epidemical DTN", Ad Hoc Networks, vol. 16, (2014), pp. 197-209.

[4] A. Goldsmith, "Wireless Communications", Cambridge University Press, Reprinted, (2007).

[5] S. Tanachaiwiwat and A. Helmy, "On the performance evaluation of encounter-based worm interactions based on node characteristics", Cornell Univ., Ithaca, NY, USA, Tech. Rep. (2007).

[6] J. P. Maranhão, V. Rolla, M. Curado and R. Salles, "A Model for Epidemical DTN Considering Effects of Path Loss and Interference", IEEE Communications Letters, (2014).

[7] J. Turkka and M. Renfors, "Path loss measurements for a non-line-of-sight Mobile-to-Mobile environment", Proceedings of the International Conference on Intelligent Transport System Telecommunications, Phuket, Thailand, (2008).

[8] F. Zhang, I. Joe and P. YongJin, "A Link Reliability and Distance Based Multipath Protocol for MANETs", 07 Ad Hoc \& Wireless Sensor Network, (2012).

[9] F. Zhang, J. Ma, D. Gao, X. Tan and Y. Liu, "A protocol for delay tolerate network", International Conference on Engineering Technology and Application, ICETA, (2015).

[10] Q. Wang and Z. Haas, "Performance Analysis of Epidemic Routing for Delay-Tolerant Networks", Resource Management in Mobile Computing Environments Modeling and Optimization in Science and Technologies, vol. 3, (2014).

[11] Fuquan Zhang, Inwhee Joe, Demin Gao, Yunfei Liu, "Measurement and Application of Link Reliability for Wireless Mobile Networks" International Journal of Multimedia and Ubiquitous Engineering, vol. 11, no. 6, (2016).

[12] F. Zhang, K. Chen, D. Gao , Y. Liu and I. Joe, "A Model for Epidemical Delay Tolerate Networks Considering Link Reliability", The 8th International Conference on Multimedia, Computer Graphics and Broadcasting, (2016).

[13] The Network Simulator", http://www.isi.edu/nsnam/ns

\section{Authors}

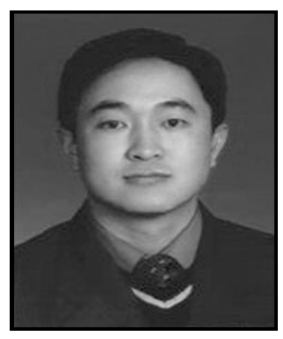

Zhang fuquan, he received M.S degree in Computer Science from Shen Yang Li Gong Univ. in 2005, and Ph.D. degree in Hanyang University, Seoul, Korea. His research fields include 3G/4G cellular systems and wireless mesh network.

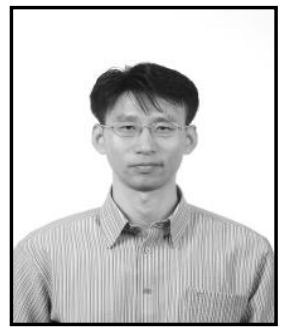

Inwhee Joe, he received B.S. and M.S. degrees in Electronics Engineering from Hanyang University, Seoul, Korea, and Ph.D. degree in Electrical and Computer Engineering from Georgia Institute of Technology, Atlanta, GA in 1998. Since 2002, he has been a faculty member in the Division of Computer Science \& Engineering at Hanyang University, Seoul, Korea. His current research interests include wireless sensor networks, 3G/4G cellular systems, mobility management, multimedia networking. 


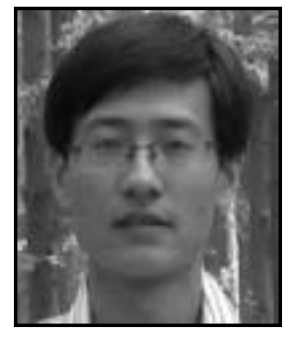

Demin Gao, he received a Ph.D. degree in 2012 from Nanjing University of Science and Technology Department of Computer Science and Engineering. In 2012, he joined in the Nanjing Forestry University as a lecturer. His research interests include routing protocols for delay tolerant, data aggregation and multiconstrained routing algorithms in wireless sensor networks and wireless sensor networks.

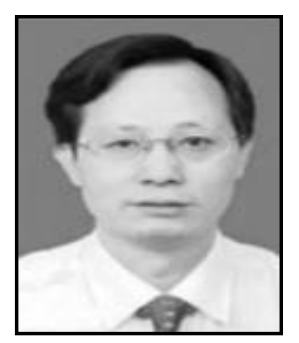

YunFei Liu, he received a Ph.D. degree from Nanjing University of Aeronautics \& Astronautic of China in 2005. He is a professor of Electronic and Communication Engineering at the College of Information Science and Technology, Nanjing Forestry University. His current research interests is signal processing technology. 
International Journal of Future Generation Communication and Networking Vol. 9, No.10, (2016) 